\title{
Recognition and Classification of Ancient Dwellings based on Elastic Grid and GLCM
}

\author{
Yang-Fan, Shen Lai-xin \\ School of Information Engineering, HuangShan University, \\ Anhui 245041, China \\ Electronics and Information Engineering School, Tongji University, \\ Shanghai 201804, China
}

\begin{abstract}
Rectangle algorithm is designed to extract ancient dwellings from village satellite images according to their pixel features and shape features. For these unrecognized objects, we need to distinguish them by further extracting texture features of them. In order to get standardized sample, three pre-process operations including rotating operation, scaling operation, and clipping operation are designed to unify their sizes and directions. According to the partition ideas of Elastic Grid, we can choose some characteristic rows and columns of an image, and the intersecting of them form some grids, which can depict the local features of the image. Five approximate independent statistics of GLCM (Gray Level Co-occurrence Matrix) of each grid can obtain and fusion a value by using Lebesgue measure, and all the value of all grids can combine into a vector, which can reflect local features and global features of the object. We utilize classification algorithm based on GLVQ Neural Network to recognize and classify all extracted objects, and get a 3-layers classification tree. Thus, we can implement the recognition and classification of different objects and the chronology classification of different dwellings. Experiments show that the correct extracted rate of dwellings is about $76.3 \%$ by using rectangle algorithm, and the chronology classification rate of ancient dwellings is more than $88.9 \%$ by using the feature-extracting algorithm based on extended Elastic Grid and GLCM.
\end{abstract}

Keyword: satellite image, GLCM, Elastic Grid, GLVQ, chronology recognition

\section{Introduction}

Huizhou area has thousands of years of ancient architecture resources that called "Old Ten" including ancient dwelling, ancient ancestral temples, ancient memorial archways, ancient trees, ancient bridges, ancient dams, ancient wells, ancient ponds, ancient water gaps and ancient pagodas [1]. The distributions of these ancient architectures are scattered, which lead to the concentrated protecting difficult and time-consuming, and their real-time monitoring and status analysis are difficult to achieve. With the wide applications of civilian satellite image, according to the latitude and longitude of these ancient architectures, we can quickly extract their object images from satellite images, and further carry out feature extraction and rapidly complete the accurate recognition of them. Finally, we can achieve accurate positioning, real-time state detection, state changed analysis, accurate recognition and classification of them.

In this paper, our target is ancient dwellings and ancient villages. According to the latitude and longitude ranges of ancient villages, the corresponding satellite images of them can be downloading from 18-levels Google Satellite Maps using the API function provided by Google Earth. Based on pixel features and shape features of the ancient dwellings, we can extract their object images from the ancient villages. In order to 
eliminate those possible wrong recognized objects, we further extract their texture features to characterize and classify them. Here, In order to get the local features of these extracted objects, we use Elastic Grid to partition them. For each grid, we can extract GLCM statistics such as auto correlation, contrast, entropy, clustering shape information measure. The five statistics are independent and obey the Lebesgue Measure rule, and they can fusion a feature value. All these values of all grids can form a feature vector to represent the local and global features of the object. Using the distance computing of vectors and the minimum generalization LVQ neural network (GLVQ) classification algorithm, we can construct a 3-levels classification tree of all extracted objects, and get accurate recognition and chronicle classification of ancient dwellings.

\section{[1] Research Status}

The mining and application of satellite images is becoming more and more popular [2-

5]. In [2], the authors proposed a method to generate the land-sea boundary template, which contained the generation of aim grid, choosing the world coastline database and the search algorithm. In [3], the authors proposed a low dimensional estimation method of fluid motion based on Helmholtz decomposition of motion field, and applied the satellite image data to the large range of wind reconstruction. In [4], the authors used Gabor textures and GLCM statistics to segment an image in multiple scales and obtain the regions of interest of bridge. In [5], the authors used PCA and GLCM texture features, which extend morphological profile and part spectral features to classify hyper-spectral image.

There are some methods to extract local features by using wavelet [6], SIFT [7], Gabor $[4,8]$. In [6], the authors used the improved orthogonal matching wavelet algorithm to realize typhoon feature extraction in the NWP. In [7], the authors proposed a fingerprint retrieval method of multi-feature fusion based on Ada-Boost to improve the robust of video fingerprint, and they gain the weight of SIFT, temporal and audio features adaptively. In [8], the authors combined the Gabor transform with the hierarchical histogram to extract facial expressional features, which could hierarchically represent the change of texture in local area.

There are some methods extracting multi- features and fusion methods $[9,10]$. In [9], the authors proposed the methods of shot similarity measure based on probabilistic distance and fusing temporal \& spatial features. They calculated the temporal-similarity by the intersection of shot weight color histogram, and spatial-similarity by probabilistic distance of spatial features in high dimension space. In [10], the authors used probabilistic neural networks and Dezert-Smarandache theory to realize multi-feature fusion recognition of image targets.

There are some methods extracting local features by using partitioning ideas. In [11], the authors used strokes densities in elastic mesh region and the directional feature decomposition in its local features of Uyghur word, and extracted the features of the strokes of cross-points, rings and arcs as well as the additional strokes and peripheral contours in its global features. In [12], the authors proposed new feature extraction approach based on double elastic mesh and fuzzy feature by combining the double elastic mesh technology and the fuzzy feature extraction approach of Chinese characters. In [13], the authors used face structure-based region partitioning method to divide the face into several regions, and used elastic graph with different density and deforming scale to perform the face matching process.

There are some methods extracting global features from multimedia by using PCA [5, 14], GLCM [5, 15-21], GMM [15], et al. In [14], the authors constructed a decision tree classification based on PCA and multi-scale texture, which can extract the types of ground objects effectively. In [15], the authors adopt GLCM to extract plate texture feature and estimated the parameters about the GMM combining with K-Means algorithm. In [21], 
the authors analyzed the influence law of parameters (direction, distance, gray scale, window size) on GLCM texture features of color image.

The recognition and classification of objects usually adopt Ada-Boost [8], LVQ (Learning Vector Quantization Neural Network) [22], GLVQ (Generalized LVQ) [23], decision tree [24] and SVM (Support Vector Machine) [25], et al. In [22], a novel signal processing method based on EMD and LVQ is proposed and applied in the field of biological signal processing, and the results are compared with the SVM and error back propagation neural network classification algorithm. In [23], a measure function of vector similarity with feature data range taken into account is proposed, and GLVQ-FR is put forward. In [24], the authors proposed a zero-watermarking model for relational database, which is based on improved $\mathrm{C} 4.5$ algorithm in combination with general zero-watermarking model of relational database. In [25], the authors made use of the advantages of SVM and LDA, and used LDA topic model for modeling and feature selection in order to determine the number of hidden topic number and topic-document matrix.

This paper extracts the objects from the village satellite images, and then makes division of the object images based on the improved Elastic Grid to obtain a number of disjoint sub-grids. For each grid, we extract the GLCM's 5-weak correlation statistics, and fusion the feature values by using Lebesgue measure, and combines all value of grids into a vector, which represents the objects fingerprint. We use GLVQ classification algorithm to construct a 3-level classification tree, which can complete the accurate recognition and classification of ancient dwellings.

\section{Dwelling Objects Extracting Algorithm based on Pixel Features and Shape Feature}

Extracting algorithm is to look up some rectangular windows, which satisfy gray value features and shape features of ancient dwellings from village satellite images. Firstly, village images need to extract according their longitudes and latitudes from Google satellite map. Secondly, objects' gray value interval are set to $[65,160]$, and the gray value of non-objects area are set to zero, and then we can exclude these impossible object area, such as rivers, roads, grass and forests, but there are still some wrong extraction, which need to be excluded by using further texture features.

We give the method of extracting rectangular windows. First, we look for a line with continuous pixels. if its size is equal to the width of dwelling sample, we consider the line is a short edge. Second, we look up another line with continuous pixels in the vertical direction of the short edge. If its size is equal to the height of dwelling sample, we consider the line as a long edge of dwelling. Third, we use area method to judge the rectangle surround with the short edge and long edge. If the number of pixels of the rectangle is above $75 \%$ size of standard dwelling sample, we consider the rectangle as a dwelling object and extract its image according to its position and size. Here, we may need to alter the width and length according to the corresponding length of continuous pixels. Extraction algorithm is shown in Figure 1. 


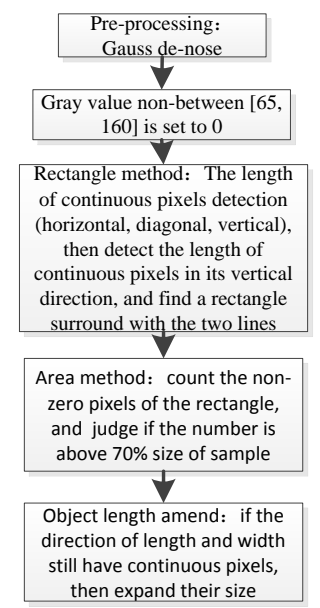

Figure 1. The Extracting Algorithm of Dwelling Objects

In the extracting algorithm, it is necessary to pre-set the length and width of the extraction objects. The size of pre-set is influenced the detection results. If the size is set too small, a bigger dwelling may be consider as two dwelling objects. If the size is set to too large, some litter objects may be omitted. Meanwhile, the size of many dwellings are not same, so we need to expand the correspond size of the objects according its number of continuous pixels.

For these extracting objects, we use the preprocessor operators to standardize them (direction and size), and then use partition technology based on improved Elastic Mesh to divide the object images and obtain their local features.

\section{Objects' Pre-processing and Elastic Grid Partition Method}

Due to the different distributions and directions of dwellings in the villages, the extracted rectangular objects have different sizes and directions. Their edges or corners are blank. We need to standardize the objects in order to extract accurate texture feature, make the long edge horizontal, and eliminate the effect of blank boundary. We have designed three pre-processing operators including rotation operator, scaling operator and cutting operator.

(1) Rotation operator: the long side of the detection objects the horizontal angle $\boldsymbol{\theta}$, and I use imrotate function according to the counter clockwise rotation, i.e. imrotate (I, $\theta)$.

(2) Scaling operators: the ratio of the size of the current object to the size of the standard sample is $\boldsymbol{\alpha}$, using the bilinear difference algorithm, and unify the size of objects, i.e. bilinear $(I, \alpha)$.

(3) Cutting operator: calculate the starting point $\mathrm{s}(\boldsymbol{x}, \boldsymbol{y})$ and the standard length $w$ of the object, and use imcrop function to extract the object' sub-region, i.e. imcrop $(I, s, w)$.

We process all extracted objects by using these pre-processing operators to obtain standard object images, and then we use Elastic Grid (EG) technology to partition them.

Here, I $(x, y)$ is the image matrix, its size is $\boldsymbol{M} * \boldsymbol{N}$, and $\boldsymbol{I}_{\boldsymbol{k}}, \boldsymbol{I}_{\boldsymbol{i}}$ are any two rows, which satisfied formula (1). $\boldsymbol{J}_{\boldsymbol{i}}, \boldsymbol{J}_{\boldsymbol{k}}$ are any columns, which satisfied formula (2):

$$
\begin{aligned}
& \int_{I_{i}}^{l_{t+1} N} \int_{1}^{N} I(x, y) d x d y=\int_{I_{k}}^{I_{k+1}} \int_{1}^{N} I(x, y) d x d y=\frac{1}{N_{1}} \int_{1}^{M} \int_{1}^{N} I(x, y) d x d y \\
& \int_{1}^{M} \int_{J_{i}}^{J_{I_{1}+1}} I(x, y) d x d y=\int_{1}^{M} \int_{J_{k}}^{J_{k+1}} I(x, y) d x d y=\frac{1}{N_{2}} \int_{1}^{M} \int_{1}^{N} I(x, y) d x d y
\end{aligned}
$$

Because the gray values of the extracted objects are unevenly distributed, it is difficult to fully conform to the formula (1) and (2), so we need to modify them. The formula (1) is 
altered to the following formula (3), and the variant of formula (2) is omitted.

$$
\begin{aligned}
& \frac{1}{N_{1}+1} \int_{1}^{M} \int_{1}^{N} I(x, y) d x d y \leq \int_{I_{i}}^{I_{i t}} \int_{1}^{N} I(x, y) d x d y \\
& =\int_{I_{k}}^{I_{k+1}} \int_{1}^{N} I(x, y) d x d y \leq \frac{1}{N_{1}-1} \int_{1}^{M} \int_{1}^{N} I(x, y) d x d y
\end{aligned}
$$

According to the above formulas, we use Elastic Grid to partition objects into several dis-conjoint sub-grids. The traditional Elastic Grid technique uses the mean of all the pixel gray value of the sub-grid to characterize the grid. Sometimes, the mean value cannot character the uniqueness of irregular dwellings, so we use a five approximate independent statistics of GLCM to represent the texture features of each sub-grid.

\section{GLCM Statistics Analysis}

GLCM (Gray Level Co-occurrence Matrix) reflects the joint distribution of gray values of neighboring points on an image, and it is the brightness change of second order statistical properties of the image. An image' GLCM concerning direction $\theta$ and distance $\mathrm{d}$ is defined as following: $\phi \in\{0,45,90,135\}, d \in\{1,2, \ldots \mathrm{N}\}$,

$P(i, j, \theta, d)=\#\left(f\left(x_{1}, y_{1}, \theta, d\right)=i, f\left(x_{2}, y_{2}, \theta, d\right)=j \mid\left(x_{1}, y_{1}, \theta, d\right),\left(x_{2}, y_{2}, \theta, d\right) \in M \times N\right.$

Function $\#(x)$ is defined as the number of $x$. GLCM matrix reflects any two pixels' joint probability in one direction and a distance of distribution, which can characterize the texture of the image.

GLCM has totally 18 feature statistics. Many feature values usually are statistical value in physical or math meanings, such as Entropy, Angular Second Moment (ASM, also called Energy), Contrast, Cluster Shade, and Information Measure of Correlation, et al. The correlations between these statistics are relatively large, such as Energy and Entropy, Contrast and Dissimilarity, Cluster Prominence and Cluster Shape, Autocorrelation and Correlation, et al. In the process of feature selection, we should choose less relevant statistics to characterize the object texture.

After many experiments based on some extraction objects, we analyze the correlation between all 18 feature-values, and obtain 5-classes of statistics, which are \{Autocorrelation, Correlation, Sum Average, Sum Variance $\}$, Energy, Entropy, Max Probability, Sum Entropy\}, \{Contrast, Dissimilarity, Homogeneity, Difference Variance, Difference Entropy, Information Measure of Correlation 1\}, \{Cluster Prominence, Cluster Shade\}, and \{Information Measure of Correlation 2\}. In each class, there are strong correlations between them. For any two classes, there are weak correlations between them.

We can select a characteristic value representing the class from each class and form nearly five independent statistics: autocorrelation (aut), Contrast (con), Cluster Shape (cs), Entropy (ent), Information Measure of Correction 2 (im2), their definitions are listed as formula (2) to (6):

$$
\begin{aligned}
& \text { aut }=\sum_{i=1}^{n} \sum_{j=1}^{n} i j P(i, j) \\
& \text { con }=-\sum_{i=1}^{n} \sum_{j=1}^{n}(i-j)^{2} P(i, j) \\
& c s=\sum_{i=1}^{n} \sum_{j=1}^{n}\left(i+j-\mu_{x}-\mu_{y}\right)^{3} P(i, j) \\
& \text { ent }=-\sum_{i=1}^{n} \sum_{j=1}^{n} P(i, j) \log P(i, j) \\
& \text { im2 }=\left(1-\exp \left(-2\left(H X Y_{2}-H X Y\right)\right)\right)^{1 / 2}
\end{aligned}
$$

Here, we give the correlation analysis between the 5 -statistics in Table 1. 
Table 1. Correlation of the 5-Statistics

\begin{tabular}{cccccc}
\hline correlation & aut & con & cs & ent & im2 \\
\hline aut & 1 & & & & \\
con & -0.01 & 1 & & & \\
cs & -0.16 & 0.09 & 1 & & \\
ent & 0.22 & 0.12 & 0.16 & 1 & \\
im2 & 0.18 & -0.15 & 0.07 & 0.10 & 1 \\
\hline
\end{tabular}

For each grid of an object, we use these 5-statistics (aut, con, cs, ent, im2) to characterize it. Now, we give a novel method to fusion the five-independent statistics.

According to Lebesgue measure rule ${ }^{[26]}$ : If $\mathrm{A}$ is a set of the Cartesian product of several subsets, namely $\boldsymbol{A}=\boldsymbol{I}_{1} \times \boldsymbol{I}_{2} \times \ldots \times \boldsymbol{I}_{n}$, then A is Lebesgue measurable and $\lambda(\boldsymbol{A})=\mid$ $\boldsymbol{I}_{1}|| \boldsymbol{I}_{2}|\ldots| \boldsymbol{I}_{n} \mid$, where $\left|\boldsymbol{I}_{i}\right|$ represent the length of the interval.

Therefore, the five statistics can use Lebesgue measure rule to fusion into a value, the texture feature of each grid $\boldsymbol{f g}_{\boldsymbol{i}}$ can be obtained using formula (7):

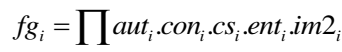

All feature values of grids of the object can combine into a multi-dimensional vector,

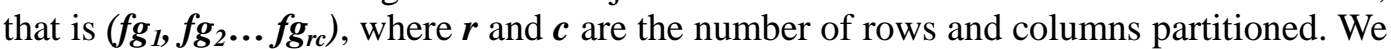
use the r.c-dimensions vector to represents the identity of the object. All the $N$ preprocessed objects have the same r.c partitions, and obtain an r.c-dimensions vector.

Based on these feature vectors of all extracted objects, we use GLVQ classification algorithm based on the minimum distance of vector and construct a 3-levels classification tree to recognize and classify them.

\section{GLVQ Classification Algorithm based on Feature Vector}

GLVQ (Generalization Learning Vector Quantization) is a kind of self-organizing neural network model with supervised learning strategies [17]. GLVQ neural network consists of three layers of neurons, namely the input layer, a hidden layer (also known as competitive layer) and an output layer.

During the training phase, we set $p$ to the first $p$-th training vectors, $T p$ of their respective class label. $C j$ is $j$-th output neuron belongs to the class label. $n$ is the number of output classes. The competition level learning process is as follows:

Step 1: Initialize the weight vector $\mathrm{W}=\left\{w_{1}, w_{2} \ldots w_{n}\right\}$, the learning rate $\eta \in[0,1]$, and the total number of iterations $\mathrm{N}$;

Step 2: For each vector $x_{p}$, do the following (1) and (2).

1)Calculate the distance $x_{p}$ with each cluster center, $\left\|\omega_{k}-x_{p}\right\|<\left\|\omega_{j}-x_{p}\right\|, j=1,2 \ldots, n$, Find winning neuron cluster centers $k$ with a minimum distance;

2)Update the weight vectors:

$W_{i j}^{\text {new }}=W_{i j}^{\text {old }}+\eta\left(x_{i}-W_{i j}^{\text {old }}\right)$, cor rect cl assi fi cat i on

$W_{i j}^{\text {new }}=W_{i j}^{\text {old }}-\eta\left(x_{i}-W_{i j}^{\text {old }}\right)$, error cl assifi cat $\mathrm{i}$ on

Step 3: Update learning rate $\eta$ :

$\eta=\eta_{0}\left(1-\frac{n}{N}\right)$

Step 4: Check the termination condition: If $n<N$ then return to Step2 else exit

GLVQ classification algorithm is actually to find the center of each class, called the prototype vectors to represent the class. Each test object and the prototype vector for each class is to calculate the distance, with the minimum distance for the object type class, when the object is classified as a certain type, this prototype vector class will be updated, 
and find new prototype vector with a minimum sum of squared distances to represent the average class.

\section{Experiments and Analysis}

We download the satellite map of the 18-levels by using the API function provided by Google Map. In this paper, we select Xi-zhou village, Shang-shan town, Xiu-ning County of Huangshan City. The village is about 180 households, with a population of 712 . The village has 2 ancient dwellings about 300 years, 19 ancient dwellings about 100 years, 4 ancient dwellings with double-patios, 12 single-patios and other dwellings without patio. Figure 2 is the satellite images of Xi-zhou village (left upper part of the village).

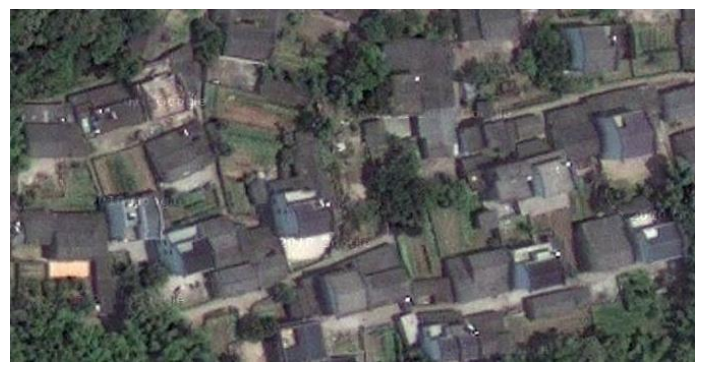

Figure 2. Part Satellite Image of Xi-zhou Village

Experiment 1: comparison of dwelling extraction algorithm

Setting the different parameters of length, width and area of filter, you can get different recognition results. Generally, all the dwellings can be identified, yet there is still some wrong identification, that is non-dwellings. When the length and width parameters of the rectangle are set as 33 and 31, and the area ratio is $70 \%$, the identification effect is shown in Figure 3:

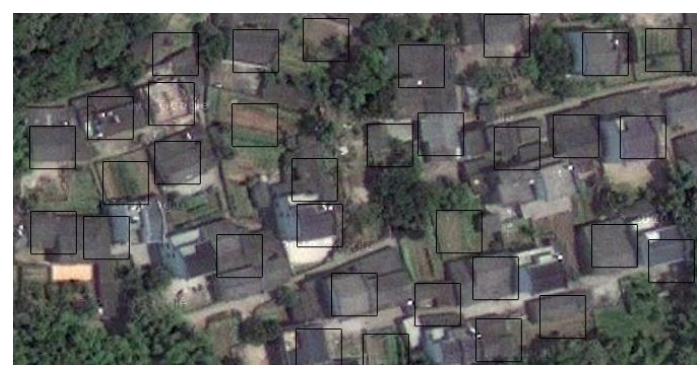

Figure 3. Extracting Result with $(33,31)$ Parameters

In the same villages, we use the sliding window method to extract dwellings [27], and the correct extracting rates by using different methods and parameters are shown in Figure 4.

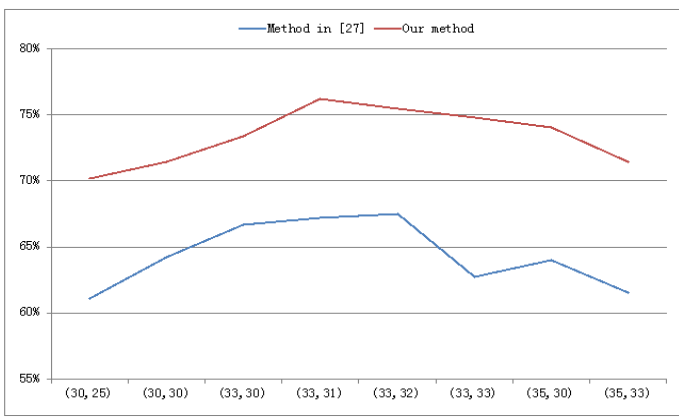

Figure 4. Extracting Rate of Different Methods and Parameters 
In fact, in [27], the authors did not look for the long edge or short edge, and only a sliding window. They find an arbitrary rectangular. When the number of the gray value of objects exceeds a certain value, they considered it as a dwelling. While we find an edge firstly, and then find a rectangle, finally we use area method to judge the rectangle. Therefore, the recognition rate of our algorithm is better.

Experiment 2 dwelling pre-processing operators

Three typical ancient dwellings (followed by double-patios, single-patio and no-patio) and the error identification of non-dwelling areas by using extracting algorithm are shown in Figure 5:

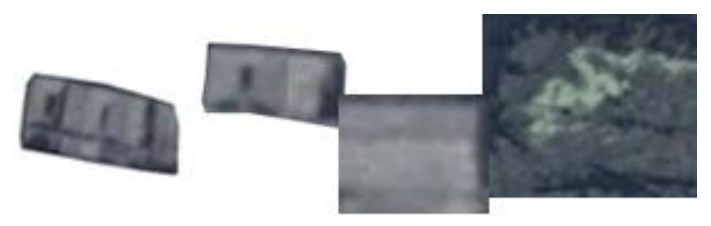

\section{Figure 5. 3-Classes Ancient Dwellings and a Wrong Recognition Object}

Using the above three pre-processing operators (rotation operator, scaling operator and cutting operator), and unify the size of the objects to $64 * 48$, and the direction is horizontal. Left to right of the upper layer is double-patio dwelling and single-patio dwelling, and the below layer are corresponding to the no-patio dwelling and wrong recognized object. The corresponding standard samples are shown in Figure 6.
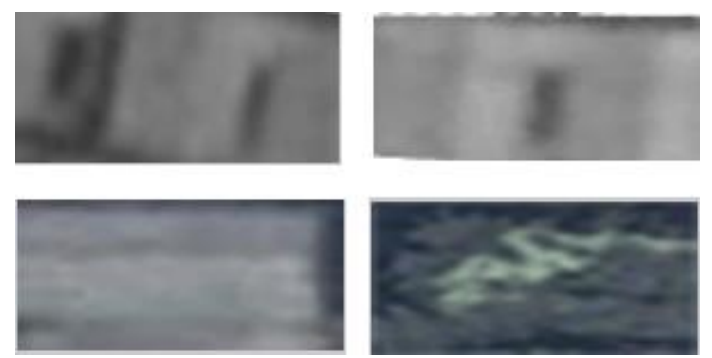

\section{Figure 6. The Standard Sample of Pre-Processed}

Experiment 3 The feature extraction and classification based on GLVQ

Ancient dwellings of Hui-zhou have rich chronicle features. The dwellings of different ages have different characteristics, especially the patio structures. In the Ming Dynasty (about 1368-1644) ancient dwellings were with double-patios structure (patio has ventilation, moisture, avoid malaria and other functions). Qing Dynasty (about 1636-1911 years) was single-patio structure. Modern dwellings were with no-patio structure. We can approximately classify all the extracted objects by using this feature. We can construct a 3-level classification tree to recognition and classify them. The first layer can recognize the dwellings and wrong recognition objects, such as dwelling class, dry grass class, hillside class, and dry pond class and so on, and then classify the dwelling class to achieve double-patios subclass, single-patio class and no-patio class. Therefore, we can achieve chronicle feature classification and recognition of the emblem of ancient dwellings.

The feature values of a grid of 3-classes ancient dwellings in Figure 6 are following.

s1 (23.96, 2.56, 0.39,-38.9, 0.89);

d1 (27.54, 2.40, 0.24,-9.4, 0.93);

w1 (22.46, 2.68, 0.36, 20.63, 0.92);

The features of the extracted objects are different in the different partitions of Elastic Grid. With the increase of the size of partitions, the feature is more abundant, and the 
recognition rate is higher. When the partition size is too big, the object-matching rate will decline. Different grid partitions will reduce different classification results of the first layer and the second layer, and the results are shown in Figure 7.

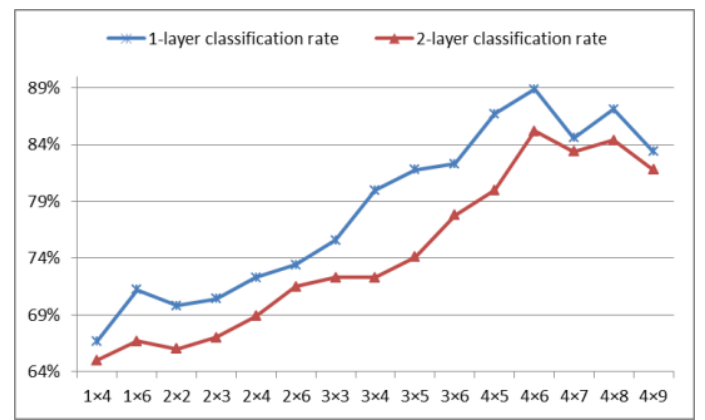

Figure 7. Classification Result of Different Grid Partitions

In Figure 7, the 1-layer classification rate characterizes the correct classification rate of all the objects, mainly for the dwelling class, the dry grass class, the hillside class and the dry pond class. The 2-layer classification rate characterizes the dwellings classification rate, mainly for double-patios class, single-patio class and non-patio class. As can be seen, when the partition is the minimum partition, namely $1 \times 1$ grid, it is equivalent to no-partition. At this time, the classification rate is the lowest. When the partition is $4 \times 6$, the classification rate is the highest. When the size is $4 \times 7$ or $4 \times 8$ partition, the classification rates have down trend.

The maximum partition is $w \times h$, at which every pixel is a grid, and the effect is relatively poor for the irregular of the object extraction, there is an over-partition of the situation. The size of $1 \times 1,4 \times 1,1 \times 6$ and $2 \times 2$ are the cases of the less-partitions.

The chronicle classification rate is the 2-layer classification result. The 2-layer classification rate compare with the paper [9] (based on the Gabor coefficients feature extraction method) and the paper [21] (feature extraction method based on GLCM with Angular Second Moment, Entropy, Contrast and Correlation) and get the results as shown in Table 2.

Table 2. 1-Layer Classification Rate with Different Methods

\begin{tabular}{cc}
\hline Method & correct rate \\
\hline Gabor [9] & $81.8 \%$ \\
GLCM [21] & $75.6 \%$ \\
Our method & $88.9 \%$ \\
\hline
\end{tabular}

\section{Conclusion}

From the Google map, we can obtain the satellite image around the world. According to the gray value feature, shape feature and texture feature of our goals, we can use distributed algorithm to extract the ancient dwellings objects from the global satellite image. Through accurate recognition and classification, we can mark their position (longitude and latitude, and the location in the village) and get their chronicle features.

By using the same method, we can identify the road from villages and deduce the development process of the road according to the location and chronicle of the ancient dwelling. When the ancient dwellings in different location and the different location of the road is obtained, the topology of the ancient villages can be reconstructed. We can establish the village drop mathematical model, which can reproduce the evolution of the ancient village and has certain scientific significance in the protection of ancient villages and dwellings. 


\section{Acknowledgement}

This work is supported by Chinese Ministry of Culture, Science and Technology Innovation Project (WHBKJCXXM20142554), Chinese State Administration of Cultural Heritage, Cultural Preservation and Technological Research (2013-YB-SQ-175), Chinese Ministry of Culture, National Culture and Science Technology Promotion Projects (GJWHKJTSXM20151991) and HuangShan University Science Research Project (2015xhwh012).

\section{References}

[1] Research Team of Huangshan Social Science League. A Project Report for Protecting 1000 Ancient Villages in Huangshan City [J]. Journal of Huangshan University, 2008, 9(4): 1-9

[2] Xu Wen, Lv Ke, Yang Lei, Lin Zheng-zong, Zhai Rui. Generation method of land-sea boundary template in geostationary satellite image registration [J]. Computer Engineering, 2014, 40(4): 154-158

[3] Chen Xiao-jing, Jing Zhong-liang, Zhang Jun. Large-scale wind field reconstruction based on satellite image data [J]. Computer Engineering, 2013, 39(12): 233-236

[4] Zhang shao-ming, Yu Sheng, Wang Jian-mei, Feng Tian-tian. Attributes estimation of bridge with TerraASR-X Imagery [J]. Journal of Tongji University (Natural Science), 2014, 42(4): 611-617

[5] Wang Zeng-mao, Du Bo, Zhang Liang-pei, Zhang Le-fei. Based on texture feature and extend morphological profile fusion for hyperspectral image classification [J]. Acta Photonica Sinica, 2014.8, 43(8): 0810001-0810008

[6] Wang Yi, Sun Jia-jun, Xiang Jie, Ren Jian-qi. Segmentation of typhoon cloud systems with orthogonal matching pursuit algorithm [J]. Acta Oceanologica Sinica, 2012.9, 34(5): 59-64

[7] Wang Fu-li, Ouyang Jian-quan. Fingerprint retrieval method of multi-feature fusion based on AdaBoost [J]. Computer Engineering, 2012.11, 38(21): 272-275

[8] Liu Xiao-min, Zhang Yu-jin. Facial expression recognition based on Gabor histogram feature and MVBoost [J]. Journal of Computer Research and Development, 2007, 44(7): 1089-1096

[9] Li Xian-hui, Zhan Yong-zhao, Ke Jia. Shot similarity measure based on probabilistic distance and temporal and spatial features fusion [J], Application Research of Computers, 2010.4, 27(4): 1526-1529

[10] Li Xin-de, Yang Wei-dong, DEZERT Jean. An airplane image target's multi-feature fusion recognition method [J]. ACTA Automatica SINICA, 2012.8, 38(8): 1298-1307

[11] Jia Jian-zhong, Gong Sheng-rong, Yimamu'aishan Abudonlikemu. Recognizing offline handwritten Uyghur text based on mixed features of Elastic mesh [J]. Computer Applications and Software, 2014.9, 31(9): 172-175

[12] Wei Wei, Guo Xiang-dan. Study of handwritten Chinese charaters' double elastic mesh and fuzzy feature [J]. Control Engineering of China, 2012.11, 19(6): 1025-1028

[13] Zhang Hai-yang, Ma Huadong. Adaptive Elastic graph face matching method in grid [J]. Journal of computer-aided design \& computer graphics, 2008.2, 20(2): 253-258

[14] Liu You-shan, Lv Cheng-wen, Zhu Fengxia, Gao Chao. Extraction of high spatial resolution remote sensing image classification based on PCA and multi-scale texture feature [J]. Remote sensing technology and application, 2012.10, 27(5): 706-711

[15] Chen Yu, Wang Ai-fei, Jiang Lu, Cao Jun. Plate Texture classification algorithm based on kmeans-GMM model [J].Journal of Harbin University of Science and Technology, 2013.8, 18(4): 69-73

[16] Liu Tian-shi, Xiao Min-min, Li Xiang-juan. Textural feature extraction algorithm fused with direction measure and GLCM method [J]. Science Technology and Engineering, 2014.11, 14(32): 271-275

[17] Jie-Sheng Wang, Xiu-Dong Ren. GLCM based extracted of flame image texture features and KPCA-GLVQ recognition method for Rotary Kiln combustion working conditions[J]. International Journal of Automation and Computing, 2014.2, 11(1): 72-77

[18] Zheng Shudan, Zheng Jianghua, Shi Minghui, et al. Classification of cultivated Chinese medicinal plants based on fractal theory and gray level co-occurrence matrix textures [J]. Journal of Remote Sensing, 2014.4, 18(4): 868-876

[19] Ren Guo-zhen, Jiang Tao. Study on GLCM-based texture extraction methods [J]. Computer Applications and Software, 2014.11,31(11): 190-325

[20] Li Ming, Lu Fang-bo, Chen Hao. Dual weighted multi-feature texture segmentation based on QWT and GLCM [J]. PR \& AI, 2014.3, 27(3): 263-271

[21] Hou Q Q, Wang F, Yan L. Extraction of color image texture feature based on gray level co-occurrence matrix [J]. Remote Sensing for Land \& Resources, 2013, 25(4): 26-32

[22] Yu Wei, Zhou Ya, Ma Jing-jing, Wan Daili, Liu Lun, Zhang Canbin. Signal feature extraction and classification method based on EMD and LVQ Neural Network [J]. Journal of Data Acquisition and Processing, 2014.9, 29(5): 683-687

[23] $\mathrm{Hu}$ Yao-min, Xiong Xin. Vehicle classification research based on improved GLVQ algorithm [J]. Computer Engineering and Applications, 2014, 50(7): 162-165 
[24] Zhou Gang, Wu Ke-ming. Research on zero-watermarking model of relational databases based on improved C4.5 algorithm [J]. Computer Applications and Software, 2015.1, 32(1): 64-67

[25] Li Feng-gang, Liang Yu, GAO Xiao-Zhi, Zenger Kai. Research on text categorization based on LDA-wSVM model [J]. Application Research of Computers, 2015.1, 32(1): 21-25

[26] Lebesgue, Henri. Sur l'intégration des fonctions discontinues. Annales scientifiques de l'École Normale Supérieure 1910, 27: 361-450

[27] Xiao Bai, Huigang Zhang, and Jun Zhou. VHR object detection based on structural feature extraction and query expansion. IEEE Transactions on Geoscience and Remote Sensing, 2014, 52(10): 6508-6520

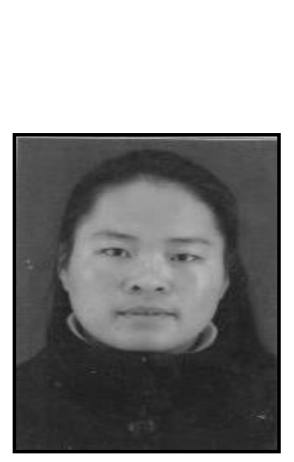

\section{Authors}

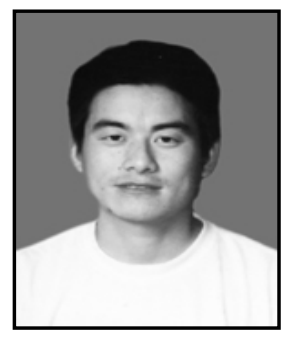

Yang fan received the M.S. degree from Hefei University of Technology, Hefei, China, in 2007. She is currently an instructor with the school of information and engineering, HuangShan University, Anhui, China. Her research interests include data mining, massive data process, and architecture. Her email is fanyang80@163.com.

Shen Lai-xin, corresponding author, received the M.S. degree from Jinan University, Guangzhou, China, in 2005. He is currently working towards the Ph.D. degree at the Department of Computer Science and Technology, Tongji University, Shanghai, China. He is also an associate professor in HuangShan University, Anhui, China. His research interests include machine learning, society computing and big data. His email is slx965@163.com. 
International Journal of Grid and Distributed Computing Vol.9, No.5 (2016) 